\title{
ORTHOGONAL EXPANSIONS WITH POSITIVE COEFFICIENTS
}

\section{RICHARD ASKEY 1}

In this note we give a simple proof of a generalization of a theorem of Szegö. The theorem in question is

$$
(\sin \theta)^{2 \lambda-1} P_{n}^{(\lambda)}(\cos \theta)=\sum_{k=0}^{\infty} \alpha_{k, n}^{\lambda} \sin (n+2 k+1) \theta
$$

where $\lambda>0, \lambda \neq 1,2, \cdots$, and

$$
\alpha_{k, n}^{\lambda}=\frac{2^{2-2 \lambda}(n+k) ! \Gamma(n+2 \lambda) \Gamma(k+1-\lambda)}{\Gamma(\lambda) \Gamma(1-\lambda) k ! n ! \Gamma(n+k+\lambda+1)}
$$

and $P_{n}^{(\lambda)}(\cos \theta)$ is given by

$$
\left(1-2 r \cos \theta+r^{2}\right)^{-\lambda}=\sum_{n=0}^{\infty} P_{n}^{(\lambda)}(\cos \theta) r^{n} .
$$

Recall that $\sin (n+1) \theta / \sin \theta=P_{n}^{(1)}(\cos \theta)$ so that (1) may be written

$$
(\sin \theta)^{2 \lambda-1} P_{n}^{(\lambda)}(\cos \theta)=\sum_{k=0}^{\infty} \alpha_{k, n}^{\lambda} P_{n+2 k}^{(1)}(\cos \theta)(\sin \theta)
$$

or

$$
(\sin \theta)^{2 \lambda} P_{n}^{(\lambda)}(\cos \theta)=\sum_{k=0}^{\infty} \alpha_{k, n}^{\lambda} P_{n+2 k}^{(1)}(\cos \theta)(\sin \theta)^{2}
$$

This suggests that a formula of the form

$$
(\sin \theta)^{2 \lambda} P_{n}^{(\lambda)}(\cos \theta)=\sum_{k=0}^{\infty} \alpha_{k, n}^{\lambda, \mu} P_{n}^{(\mu)}(\cos \theta)(\sin \theta)^{2 \mu}
$$

is true. Since $\alpha_{\boldsymbol{k}, n}^{\lambda}$ is positive for $0<\lambda<1$ we might conjecture that $\alpha_{k, n}^{\lambda \mu}$ is positive for $\lambda<\mu$. In fact, we show that it is for $(\mu-1) / 2<\lambda<\mu$. The condition $(\mu-1) / 2<\lambda$ is necessary to obtain convergence of the series (2).

This result follows from an old result of Gegenbauer [1] which has almost been forgotten by the mathematical community. Since $P_{n}^{(\mu)}(x)$ is a polynomial we may write

Received by the editors December 1, 1964.

1 Supported in part by N.S.F. grant GP-3483. 


$$
P_{n}^{(\mu)}(x)=\sum_{k=0}^{n} \beta_{k, n}^{\lambda, \mu} P_{k}^{(\lambda)}(x) .
$$

Gegenbauer gives the value of $\beta$ as

$$
\beta_{k, n}^{\lambda, \mu}=\frac{\Gamma(\lambda)(k+\lambda) \Gamma((n-k) / 2+\mu-\lambda) \Gamma(\mu+(k+n) / 2)}{\Gamma(\mu)[(n-k) / 2] ! \Gamma(\mu-\lambda) \Gamma(\lambda+1+(k+n) / 2)}
$$

if $n-k$ is even, $n \geqq k$, and $\beta_{k, n}^{\lambda, \mu}=0$ otherwise. Observe that $\beta \geqq 0$ if $\mu \geqq \lambda$. A simple proof of this is in [2].

We also need the following simple fact. Let $w(x)$ and $w_{1}(x)$ be positive functions on a set $E$. Let $\left\{p_{n}(x)\right\}$ and $\left\{q_{n}(x)\right\}$ be the orthonormal polynomials associated with $w(x)$ and $w_{1}(x)$ respectively. Then if

$$
q_{n}(x)=\sum_{k=0}^{n} c_{k, n} p_{k}(x)
$$

we have

$$
w(x) p_{k}(x)=\sum_{n=k}^{\infty} c_{k, n} q_{n}(x) w_{1}(x) .
$$

The convergence of the series can be taken in the appropriate $L^{2}$ space if $[w(x)]^{2} / w_{1}(x)$ is integrable. (5) follows immediately from (4) since the Fourier coefficients are the same in the two expansions.

Using (3), (4) and (5) and remembering that $\left\{P_{n}^{(\lambda)}(x)\right\}$ are orthogonal but not orthonormal we get

$$
\left(1-x^{2}\right)^{\lambda-1 / 2} P_{n}^{(\lambda)}(x)=\sum_{k=0}^{\infty} \alpha_{k, n}^{\lambda, \mu} P_{n+2 k}^{(\mu)}(x)\left(1-x^{2}\right)^{\mu-1 / 2}
$$

where

$$
\alpha_{k, n}^{\lambda, \mu}=\frac{\Gamma(\mu) 2^{2 \mu-2 \lambda}(n+2 k+\mu)(n+2 k) ! \Gamma(n+2 \lambda) \Gamma(n+k+\mu) \Gamma(k+\mu-\lambda)}{\Gamma(\mu-\lambda) \Gamma(\lambda) n ! k ! \Gamma(n+k+\lambda+1) \Gamma(n+2 k+2 \mu)} .
$$

Using the asymptotic formula for $P_{n}^{(\lambda)}(x)$ it is easy to see that the series in (6) converges for $-1<x<1$ if $\lambda>(\mu-1) / 2$. Also observe that $\alpha_{i, n}^{\lambda, \mu}>0$ for $(\mu-1) / 2<\lambda<\mu$.

Setting $n=0$ in (6) we get

$$
\begin{aligned}
\left(1-x^{2}\right)^{-\alpha}= & \frac{\Gamma(\mu) 2^{2 \alpha} \Gamma(2 \mu-2 \alpha)}{\Gamma(\alpha) \Gamma(\mu-\alpha)} \\
& \cdot \sum_{k=0}^{\infty} \frac{(2 k+\mu)(2 k) ! \Gamma(k+\mu) \Gamma(k+\alpha)}{k ! \Gamma(k+\mu-\alpha+1) \Gamma(2 k+2 \mu)} P_{2 k}^{(\mu)}(x) .
\end{aligned}
$$


For $\alpha=1 / 2$ and $\mu=1 / 2$ this is due to Bauer and $\alpha=1 / 2, \mu>0$ it is due to Gegenbauer [1].

There are two other instances of polynomial expansions of different orthogonal polynomials that involve the classical polynomials and have positive coefficients.

The better known one is

$$
L_{n}^{(\beta)}(x)=\sum_{k=0}^{n} \frac{\Gamma(\beta-\alpha+n-k)}{\Gamma(\beta-\alpha)(n-k) !} L_{k}^{(\alpha)}(x),
$$

where $L_{n}^{(\beta)}(x)$ are the Laguerre polynomials of order $\beta$, degree $n$, and $\beta>\alpha$. Notice that the coefficients are positive. See $[3$, p. 209] for (8).

For Jacobi polynomials, $P_{n}^{(\alpha, \beta)}(x)$, the following expansion holds

$$
\begin{aligned}
& P_{n}^{(\beta, \gamma)}(x)=\frac{\Gamma(n+\gamma+1)}{\Gamma(\beta-\alpha) \Gamma(n+\gamma+\beta+1)} \sum_{k=0}^{n} \\
& \frac{\Gamma(n+k+\beta+\gamma+1) \Gamma(n-k+\beta-\alpha) \Gamma(k+\alpha+\gamma+1)(2 k+\alpha+\gamma+1)}{\Gamma(n+k+\alpha+\gamma+2) \Gamma(n-k+1) \Gamma(k+\gamma+1)} P_{k}^{(\alpha, \gamma)}(x) .
\end{aligned}
$$

See [4, p. 254]. Again notice that, if $\gamma>-1$ and $\beta>\alpha \geqq 0$, the coefficients are positive. The common feature of both of these results is that the polynomials are normalized correctly at the right point. In the Laguerre case $L_{n}^{(\alpha)}(0)>0$ and $P_{n}^{(\alpha, \beta)}(1)>0$ for Jacobi polynomials. An interesting conjecture can be formulated of which these are a special case. For convenience we formulate it on $(0, \infty)$.

Let $w(x)$ be a positive function on $(0, \infty)$ such that $\int_{0}^{\infty} x^{n} w(x) d x$ exists for each $n=0,1, \cdots$. Let $\left\{p_{n}(x)\right\}$ be the orthonormal polynomials with respect to $w(x)$ normalized by $p_{n}(0)>0$. All of the zeros of $p_{n}(x)$ are in $(0, \infty)$ so this is possible. Let $\left\{p_{n}^{\alpha}(x)\right\}$ be the polynomials orthonormal with respect to $x^{\alpha} w(x)$ normalized in the same way. Then if $\alpha>0$ we conjecture that

$$
p_{n}^{\alpha}(x)=\sum_{k=0}^{n} \alpha_{k} p_{k}(x)
$$

with $\alpha_{k}>0$.

For $\alpha$ an integer this follows from known classical results. For if $\alpha=1$ then

$$
c_{n} p_{n}^{1}(x)=\frac{p_{n+1}(0) p_{n}(x)-p_{n}(0) p_{n+1}(x)}{x}
$$

by a theorem of Christoffel [4, Theorem 2.5]. By another theorem of Christoffel [4, Theorem 3.2.2], 


$$
\frac{p_{n+1}(0) p_{n}(x)-p_{n}(0) p_{n+1}(x)}{x}=r_{n} \sum_{k=0}^{n} p_{k}(0) p_{k}(x) .
$$

Thus

$$
p_{n}^{1}(x)=r_{n} \sum_{k=0}^{n} p_{k}(0) p_{k}(x)
$$

and $p_{k}(0), p_{n}^{1}(0)$, and thus $r_{n}$ are positive by assumption. For larger integral values of $\alpha$ the result follows by iteration.

\section{BIBLIOGRAPHY}

1. L. Gegenbauer, Zur Theorie der Functionen $C_{n}^{\nu}(x)$, Denkschriften der Akademie der Wissenschaften in Wien, Math. Naturwiss. K1. 48 (1884), 293-316.

2. L. K. Hua, Harmonic analysis of functions of several complex variables in the classical domains, Transl. Math. Monographs 6 (1963), Amer. Math. Soc., Providence, R. I.

3. E. D. Rainville, Special functions, Macmillan, New York, 1960.

4. G. Szego, Orthogonal polynomials, rev. ed., Amer. Math. Soc. Colloq. Publ. Vol. 23, Amer. Math. Soc., Providence, R. I., 1959.

UNIVERSITY OF WISCONSIN 